\title{
Games of Timing with Detection Uncertainty and Numerical Estimates
}

\author{
David B. Bednarz ${ }^{\mathrm{a}}$, Paul L. Muench ${ }^{\mathrm{a}}$, Nicholas A. Krupansky ${ }^{\mathrm{a}}$, and Bernhard von Stengel ${ }^{\mathrm{b}}$ \\ ${ }^{a}$ US Army Combat Capabilities Development Command - Ground Vehicle Systems Center, \\ 6501 E. 11 Mile Road, Warren, MI USA \\ ${ }^{\mathrm{b}}$ Department of Mathematics - London School of Economics and Political Science, Columbia \\ House COL 4.12, Houghton Street, GB-London WC2A 2AE, UK
}

\begin{abstract}
Mobility and terrain are two sides of the same coin. We cannot speak to our mobility unless we describe the terrain's ability to thwart our maneuver. Game theory describes the interactions of rational players who behave strategically. In previous work ${ }^{1}$ we described the interactions between a mobility player, who is trying to maximize the chances that he makes it from point $\mathrm{A}$ to point $\mathrm{B}$ with one chance to refuel, and a terrain player who is trying to minimize that probability by placing an obstacle somewhere along the path from A to B. This relates to the literature of games of incomplete information, and can be thought of as a more realistic model of this interaction. In this paper, we generalize the game of timing studied in the previous paper to include the possibility that both players have imperfect ability to detect his adversary.
\end{abstract}

Keywords: Game theory, game of timing, duel, silent duel, detection uncertainty, sequence form, mobility

\section{BACKGROUND}

We model this game as a silent duel with the following players: The Mobility Player choosing $x$ and the Terrain Player choosing $y$.

As before ${ }^{1}$ the payoff matrix of the game is defined by:

$$
K(x, y)= \begin{cases}L(x, y) & x<y \\ \Phi(x) & x=y \\ M(x, y) & x>y\end{cases}
$$

where:

$$
\left\{\begin{array}{l}
L(x, y)=c_{1}+a x+b y \\
\Phi(x)=c_{2}+d x \\
M(x, y)=c_{1}+e x+h y
\end{array}\right.
$$

and where: $L(x, y)$ is monotone increasing in $x$ for each $y, M(x, y)$ is monotone increasing or decreasing in $x$ for each $y^{*}, L(x, y)$ is monotone decreasing in $y$ for each $x$, and $M(x, y)$ is monotone decreasing in $y$ for each $x$.

Our detection distribution for Mobility Player is uniformly distributed over the unit interval, i.e. $F(x)=x$, $f(x)=F^{\prime}(x)$, and $x \in[0,1]$ since $F(x) \equiv \int_{0}^{x} f(s) d s$.

When only one player has detection uncertainty, it is the Mobility Player who has detection uncertainty. The Terrain Player sees the Mobility Player throughout the interval $[0,1]$. Initially at $x=0$, Terrain Player is undetected, but is detected according to the distribution function $F(x)$ by the Mobility Player. Having detected

Further author information: (Send correspondence to D.B.B.)

D.B.B.: E-mail: david.b.bednarz.civ@mail.mil,

B.v.S.: E-mail: b.von-stengel@lse.ac.uk

* Note that in a classic game of timing $M(x, y)$ is monotone increasing in $x$ for each $y$. 
the Terrain Player, the Mobility Player continues to see Terrain Player. However, Terrain Player does not know that she has been detected.

The game of timing considered here is a compound game. ${ }^{2}$ In such games, if the current state is known by both players, it may be possible to formulate a functional relation between the values at different states and determine the solution. ${ }^{3}$ However, for the game of timing considered in this paper there are two states at each $x$, either Mobility Player has detected or he has not. Only the Mobility Player knows the current state with certainty, whether or not he has detected at $x$. Since both players are not informed of the current state, they cannot attach the same worth to this state and hence cannot formulate a functional equation.

Since the players have no knowledge of each others actions, this suggests randomization, and each player might wait the full time interval. By applying rather general properties that the solution to the game of timing should possess, the authors are able to guess the optimal strategy for each player.

Based on earlier work ${ }^{1}$ we shall search for strategies for Mobility Player of the form: $w^{*}=\left(w_{\alpha}\right)$ where $w(x)$ is a mixed strategy over the interval $[\alpha, 1]$. Let Value denote the value of the game.

\section{ANALYSIS}

\subsection{Single Player Detection Uncertainty}

\subsubsection{Mobility Player}

Suppose Mobility Player uses density $w$ and detects Terrain Player at $s$. Terrain Player acts at $y \in[\alpha, 1)$. The probability that Mobility Player survives is the sum of three disjoint pieces. Let $W(s)=\int_{0}^{s} w(x) d x$. The three disjoint pieces are as follows:

1. Mobility Player detects before $y$ and Mobility Player acts before $y$

$$
\int_{0}^{y} f(s) \int_{s}^{y} \frac{w(x)}{1-W(s)} L(x, y) d x d s
$$

2. Mobility Player detects before $y$ and Mobility Player acts after $y$

$$
\int_{0}^{y} f(s) \int_{y}^{1} \frac{w(x)}{1-W(s)} M(x, y) d x d s
$$

3. Mobility Player detects after $y$ and Mobility Player acts after $y$

$$
\int_{y}^{1} f(s) \int_{s}^{1} \frac{w(x)}{1-W(s)} M(x, y) d x d s
$$

Let

$$
v(x)=w(x) \int_{0}^{x} \frac{f(s)}{1-W(s)} d s
$$

be the Mobility Player's firing density at $x$ given that neither player has fired previously, and $V(x)=\int_{0}^{x} v(y) d y$ be the probability that the Mobility Player acts in $[0, x]$ given the Terrain Player did not fire prior to $x$. Note that $v(x)=0$ for $x<a$.

Rewriting equation $(3 a)^{\dagger}$

$$
\begin{aligned}
\int_{0}^{y} f(s) \int_{s}^{y} \frac{w(x)}{1-W(s)} L(x, y) d x d s & =\int_{0}^{y} w(x) \int_{0}^{x} \frac{f(s)}{1-W(s)} L(x, y) d s d x \\
& =\int_{0}^{y} v(x) L(x, y) d x
\end{aligned}
$$

${ }^{\dagger}$ Note that $\int_{0}^{y} \int_{s}^{y} d x d s=\int_{0}^{y} \int_{0}^{x} d s d x$ 
Rewriting equation $(3 \mathrm{~b})^{\ddagger}$

$$
\begin{aligned}
& \int_{0}^{y} f(s) \int_{y}^{1} \frac{w(x)}{1-W(s)} M(x, y) d x d s \\
= & \int_{y}^{1} w(x) \int_{0}^{y} \frac{f(s)}{1-W(s)} M(x, y) d s d x
\end{aligned}
$$

Rewriting equation $(3 \mathrm{c})^{\S}$

$$
\begin{array}{r}
\int_{y}^{1} f(s) \int_{s}^{1} \frac{w(x)}{1-W(s)} M(x, y) d x d s=\int_{y}^{1} w(x) \int_{y}^{x} \frac{f(s)}{1-W(s)} M(x, y) d s d x \\
=\int_{y}^{1}\left[v(x)-w(x) \int_{0}^{y} \frac{f(s)}{1-W(s)}\right] M(x, y) d s d x
\end{array}
$$

Suppose Mobility Player uses a mixed strategy $w$ and Terrain Player uses a pure strategy $y$, where $y \in[\alpha, 1)$, then the expected payoff for Mobility Player is the sum of equations (4),(5), and (6):

$$
\begin{array}{r}
\hat{H}(y)=\int_{\alpha}^{y} v(x) L(x, y) d x+\int_{y}^{1} w(x) \int_{0}^{y} \frac{f(s)}{1-W(s)} M(x, y) d s d x \\
+\int_{y}^{1}\left[v(x)-w(x) \int_{0}^{y} \frac{f(s)}{1-W(s)}\right] M(x, y) d s d x \\
=\int_{\alpha}^{y} v(x) L(x, y) d x+\int_{y}^{1} v(x) M(x, y) d x
\end{array}
$$

Substituting equations (2), the fitted planes for the payoff function, we get:

$$
\hat{H}(y)=\int_{\alpha}^{y} v(x)\left(c_{1}+a x+b y\right) d x+\int_{y}^{1} v(x)\left(c_{1}+e x+h y\right) d x
$$

Next we invoke the principle of strategies identically equal to $v$. The strategy $w$ will be optimal if the expected payoff to the Mobility Player is constant for each $y \in[\alpha, 1)$. Next we need to select $v(x)$ so that $\hat{H}(y)$ is constant for each $y \in[\alpha, 1)$. Then derive $w(x)$ from $v(x)$.

Taking the derivative of equation (8):

$$
\begin{aligned}
\hat{H}^{\prime}(y) & =v(x)\left(c_{1}+a y+b y\right)+b \int_{0}^{y} v(x) d x-v(y)\left(c_{1}+e y+h y\right)+h \int_{y}^{1} v(x) d x \\
& =v(y)\left(c_{1}+a y+b y\right)+b V(y)+h V(1)-h V(y) \\
& =v(y)\left(c_{1}+a y+b y\right)+b V(y)+h-h V(y)
\end{aligned}
$$

where $V(1)=1$. By Leibniz's rule,

$$
\hat{H}^{\prime}(y)=(a-e+b-h) y v(h)+(b-h) V(y)+h
$$

Let $k=(a-e+b-h)$ and $j=b-h$, and set $\hat{H}^{\prime}(y)=k y v(y)+j V(y)+h=0$. The solution to the resulting differential equation is

$$
V(y)=\frac{h}{j} \alpha^{\frac{j}{k}} y^{-\frac{j}{k}}-\frac{h}{j}
$$

\footnotetext{
${ }^{\ddagger}$ Note that $\int_{0}^{y} \int_{y}^{1} d x d s=\int_{y}^{1} \int_{0}^{y} d s d x$

${ }^{\S}$ Note that $\int_{y}^{1} \int_{s}^{1} d x d s=\int_{y}^{1} \int_{y}^{x} d s d x$
} 
and

$$
v(y)=V^{\prime}(y)=-\frac{h}{k} \alpha^{\frac{j}{k}} y^{-\left(1+\frac{j}{k}\right)}
$$

Recalling that $F(x)=x$ and noting that $\frac{w(x)}{1-W(x)}$ is the conditional density of acting at $x$, given detection prior to $x$, one can show that

$$
\frac{w(x)}{1-W(x)}=\frac{v(x)}{F(x)-V(x)}=\frac{v(x)}{x-V(x)} .
$$

Let

Substituting yields

$$
R(x)=\frac{w(x)}{1-W(x)}
$$

$$
R(x)=\frac{-h j \alpha^{\frac{j}{k}} x^{-\left(1+\frac{j}{k}\right)}}{k j x-k h \alpha^{\frac{j}{k}} x^{-\frac{j}{k}}-k h}, \text { where } x \in[\alpha, 1) .
$$

By equation (13) we have

$$
W(x)=1-\exp \left[-\int_{\alpha}^{x} R(s) d s\right]
$$

and

$$
w(x)=R(x) \exp \left[-\int_{\alpha}^{x} R(s) d s\right] \text { for } x \in[\alpha, 1) .
$$

We want to find the value for $\alpha$. Let $j=b-h$. From equation (11) we have

$$
V(1)=\frac{h}{j} \alpha^{\frac{j}{k}}-\frac{h}{j}=1 .
$$

Solving for $\alpha$, we obtain

$$
\alpha=\left(\frac{j+h}{h}\right)^{\frac{k}{j}}=\left(\frac{b}{h}\right)^{\frac{k}{j}} .
$$

We must have $\alpha \in[0,1]$ and the interval $[\alpha, 1]$ should be such that $V(\alpha)=0, V(x)<F(x)$ for $x \in(\alpha, 1)$, and $V(1)=F(1)=1$. If these conditions are not met, the optimal strategy for the Terrain Player is to act at $x=0$ and the Mobility Player acts when he first detects the Terrain Player. The value of the game in this case is

$$
\begin{aligned}
\text { Value } & =\int_{0}^{1} M(x, 0) f(x) d x \\
& =\int_{0}^{1}\left(c_{1}+e x\right) d x=c_{1}+e \int_{0}^{1} x d x \\
& =c_{1}+\frac{e}{2} .
\end{aligned}
$$

If there is a density part to the optimal strategy, the value of $\hat{H}(y)$ is constant for each $y \in[\alpha, 1)$ which is equal to the value of the game. Setting $y=\alpha$, the value of the game is

$$
\hat{H}(\alpha)=\int_{\alpha}^{1} v(x)\left(c_{1}+e x+h \alpha\right) d x
$$

if $\alpha$ exists.

Now we have the following equation for the Mobility Player:

$$
\hat{H}(y)=\int_{\alpha}^{y} v(x)\left(c_{1}+a x+b y\right) d x+\int_{y}^{1} v(x)\left(c_{1}+e x+h y\right) d x=v
$$

Next we need to find a similar equation for the Terrain player. Based on our previous work, ${ }^{1}$ we shall look for strategies for the Terrain player of the form $z^{*}=\left(z_{\alpha}, P\right)$ where $z(y)$ is a mixed strategy over $[\alpha, 1)$ with a point mass of weight $P$ at $y=1$. 


\subsubsection{Terrain Player}

Let us determine the optimal strategy for the Terrain player.

Suppose Terrain uses density $z$ and Mobility detects at $s$ for $s<1$, let $x(s)$ where $x(s) \in[s, 1)$ be the time Mobility will fire. The density part of the payoff to Mobility is the sum of two disjoint parts.

1. The probability that Terrain Player acts before Mobility Player. Note that $K(x, y)=M(x, y)$ for $x>y$.

$$
\int_{0}^{1} f(s) \int_{\alpha}^{x(s)} z(y) M(x(s), y) d y d s
$$

2. The probability that Terrain Player acts after Mobility Player. Note that $K(x, y)=L(x, y)$ for $x<y$.

$$
\int_{0}^{1} f(s) \int_{x(s)}^{1} z(y) L(x(s), y) d y d s
$$

At $y=1$ the payoff equals $P L(x(s), 1)$. Suppose that Terrain Player uses a mixed strategy $z$. A pure strategy for Mobility Player is when detection occurs at $s$ and Mobility Player acts at $x(s) \in[\alpha, 1)$ Then the expected payoff, $\hat{G}(x)$, is the sum of $(21)$ and (22)

$$
\begin{aligned}
\hat{G}(x) & =\int_{0}^{1} f(s) \int_{\alpha}^{x(s)} z(y) M(x(s), y) d y d s \\
& +\int_{0}^{1} f(s) \int_{x(s)}^{1} z(y) L(x(s), y) d y d s+P L(x(s), 1) .
\end{aligned}
$$

We want to find $z$ such that $\hat{G}$ is constant for $x \in[\alpha, 1]$. We want to find $z$ such that the coefficient of $f(s)$ is constant for each $s$. This is seen by considering the coefficient as a function of the upper and lower limits and taking the derivative with respect to these limits and set equal to zero.

Let

$$
\tilde{G}=\int_{\alpha}^{x} z(y) M(x, y) d y+\int_{x}^{1} z(y) L(x, y) d y+P L(x(s), 1) .
$$

By Leibniz's rule

$$
\begin{aligned}
\tilde{G}^{\prime}=z(x) M(x, x) & +\int_{\alpha}^{x} \frac{\partial}{\partial x} z(y) M(x, y) d y \\
& -z(x) L(x, x)+\int_{x}^{1} \frac{\partial}{\partial x} z(y) L(x, y) d y+\frac{\partial}{\partial x} P L(x, 1)
\end{aligned}
$$

Substituting the planar curve fit for $K(x, y)$ and setting equation (25) to zero, we find

$$
z(x)\left(c_{1}+e x+h x\right)+e \int_{\alpha}^{x} z(y) d y-z(x)\left(c_{1}+a x+b x\right)+a \int_{x}^{1} z(y) d y+P a=0 .
$$

Grouping like coefficients of $x$

$$
z(x) x(e+h-a-b)+e Z(x)+a(Z(1)-Z(x))=0 .
$$

Note that $Z(1)=1-P$.

Let $p=(e+h-a-b)$ and $q=(e-a)$, we find

$$
z(x) p x+q Z(x)+a=0 .
$$


This is the same form of differential equation that we had for the Mobility Player, where the solution is

$$
Z(y)=\frac{a}{q} \alpha^{\frac{q}{p}} y^{-\frac{q}{p}}-\frac{a}{q}
$$

and

$$
z(y)=-\frac{a}{p} \alpha^{\frac{q}{p}} y^{-\left(1+\frac{q}{p}\right)}
$$

Thus the coefficient is constant for each $s$ when we use the above $z(y)$. Hence, for $z(y)$ determined by equation (30) and $Z(\alpha)=0, \hat{G}(x)$ is independent of the function $x$ as long as $x(s) \in[\alpha, 0)$ for $s \in[0,1)$. Also, $P=1-Z(1)$ is the point mass at $y=1$.

The optimal strategy of the Terrain Player must maintain a threat of action until the very end. This behavior is not surprising because of the unequal information patterns available to both players.

\subsection{Detection Uncertainty for Both Players}

Let us modify the model of the previous section to the case where each player has imperfect ability to perceive their opponent. Let $F(x)$ be the detection distribution for the Mobility Player and $Q(y)$ be the detection distribution for the Terrain Player, where $F^{\prime}(x)=f(x), Q^{\prime}(y)=q(y)$, and $F$ and $Q$ are uniformly distributed.

\subsubsection{Mobility Player}

Suppose the Mobility Player uses density $w$ and detects the Terrain Player at $s$. The Terrain Player detects the Mobility Player at $t$ and acts at $y(t)$, where $y(t) \in[t, 1)$. The expected payoff for the Mobility Player using a similar argument as in equations (3a) through (7) in Section 2 is:

$$
\begin{aligned}
\tilde{H}(y) & =\int_{0}^{1} q(t) \int_{\alpha}^{y(t)} v(x) L(x, y(t)) d x d t \\
& +\int_{0}^{1} q(t) \int_{y(t)}^{1} v(x) M(x, y(t)) d x d t \\
& =\int_{\alpha}^{y(t)} v(x) L(x, y(t)) d x+\int_{y(t)}^{1} v(x) M(x, y(t)) d x .
\end{aligned}
$$

We want to find $v$ such that $\tilde{H}$ is constant for each $y \in(\alpha, 1)$. Using a similar argument as when deriving equations (8) through (16) we have:

$$
V(x)=\frac{h}{j} \alpha^{\frac{j}{k}} x^{-\frac{j}{k}}-\frac{h}{j}
$$

and

$$
v(x)=V^{\prime}(x)=-\frac{h}{k} \alpha^{\frac{j}{k}} x^{-\left(1+\frac{j}{k}\right)}
$$

and

$$
w(x)=R(x) \exp \left[-\int_{\alpha}^{x} R(s) d s\right], \text { for } x \in[\alpha, 1) .
$$

Using (17) we obtain

$$
\alpha=\left(\frac{b}{h}\right)^{\frac{k}{j}}
$$

If we do not have (a) $\alpha \in(0,1)$, (b) $V(1)=1$, and (c) $x<V(x)$ for $x \in[\alpha, 1)$, then the optimal strategy for both players is to act when they detect their opponent.

The value of the game is

$$
\int_{0}^{1} \int_{x}^{1} L(x, y) d y d x+\int_{0}^{1} \int_{y}^{1} M(x, y) d x d y=\frac{a}{6}+\frac{b}{3}+c+\frac{e}{3}+\frac{h}{6}
$$

If (a), (b), and (c) hold, we proceed to the Terrain Player. 


\subsubsection{Terrain Player}

Suppose the Terrain Player uses density $z$ and detects the Mobility Player at $t$. The Mobility Player detects the Terrain Player at $s$ and acts at $x(s)$, where $x(s) \in[s, 1)$. The expected payoff for the Mobility Player is:

$$
\begin{aligned}
\tilde{G} & =\int_{0}^{1} f(s) \int_{\alpha}^{x(s)} u(y) M(x(s), y) d y d s \\
& +\int_{0}^{1} f(s) \int_{x(s)}^{1} u(y) L(x(s), y) d y d s
\end{aligned}
$$

where

$$
u(y)=z(y) \int_{0}^{y} \frac{q(s)}{1-Z(s)} d s .
$$

Again we want to find $u(y)$ such that $\tilde{G}$ is constant for each $x \in[\alpha, 1)$. Using similar arguments as when deriving equations (8) through (16) we have:

$$
U(y)=\frac{a}{l} \alpha^{\frac{l}{k}} y^{-\frac{l}{k}}-\frac{a}{l}
$$

and

$$
u(y)=U^{\prime}(y)=-\frac{a}{k} \alpha^{\frac{l}{k}} y^{-\left(1+\frac{l}{k}\right)}
$$

where $l=e-a$.

Substituting the value for $\alpha$ in (35) into (39), we have $U(\alpha)=0$.

If we do not have (a) $U(1)=1$, and (b) $y<U(y)$ for $y \in[\alpha, 1)$, then the optimal strategy for both players is to act when they detect their opponent.

If $\alpha \in(0,1), V(1)=1, x<V(x), x \in[\alpha, 1), U(1)=1, y<U(y)$ for $y \in[\alpha, 1)$ then the optimal strategy of the Mobility Player is:

1. If detection occurs at $t<\alpha$ then he acts according to $v(x)$.

2. If detection occurs at $t \in[\alpha, 1)$ then he acts according to the density

$$
\frac{v(x)}{1-V(t)} \text {. }
$$

3. If detection occurs at $t=1$ then he acts at 1 . The optimal strategy for the Terrain Player is similar. To find the value of the game in this case we set $y=\alpha$ in equation (31) and we get:

$$
\text { Value }=\int_{\alpha}^{1} v(x) M(x, \alpha) d x .
$$

It is interesting to note that in this special case the value of the game is the same as equation (19), which means that in this special case the fact that the Terrain Player has detection uncertainty does not affect the value of the game.

\section{ANALYTIC EXAMPLES}

\subsection{Example 1: The role of detection uncertainty on our original game}

Considering the value of the game to the Mobility Player we have the following cases:

1. No detection uncertainty, Value $=-0.0466 .^{1}$ 
Table 1. Table of coefficients for the planes in (2)

\begin{tabular}{l|c|r}
$L(x, y)$ & $\Phi(x, y)$ & $M(x, y)$ \\
\hline$c_{1}=0.01358$ & $c_{2}=0.01368$ & $c_{1}=0.01358$ \\
$a=0.0341$ & $d=-0.03868$ & $e=-0.14037$ \\
$b=-0.0736$ & & $h=-0.05409$
\end{tabular}

2. Detection uncertainty in the Mobility Player only, Value $=-0.0566$.

3. Detection uncertainty in both players, Value $=-0.0611$.

We examined case 1 in previous work. ${ }^{1}$

We examined case 2 in previous work, ${ }^{4}$ where we saw the Mobility Player is at a disadvantage since he cannot act until he detects. The optimal strategy for the Terrain Player is to act at $x=0$ and the Mobility Player acts when he first detects the Terrain Player. By equation (18), the value of the game is: Value $=-0.0566$.

In case 3, we see the Mobility Player is at a further disadvantage because when he detects, he is not sure if the Terrain Player has detected him or not. Thus, there is even more uncertainty for the Mobility Player in this case. The optimal strategy for both players is to act when they detect their opponent.

\subsection{Example 2: Detection uncertainty on a slightly different example}

For this example, we wanted to have a density part, so we use the values from Table 1, except we replaced $b=-0.17$. In this case, $V(x)$ is less than $F(x)$ for $x \in[\alpha, 1)$. Thus, we have an optimal strategy of each player composed of a density with support on the interval $[\alpha, 1]$. The point mass for the terrain player is $P=0.8394$. By equation (17), $\alpha=0.5607$. By equation (18), the value of the game is: Value $=-0.1291$.

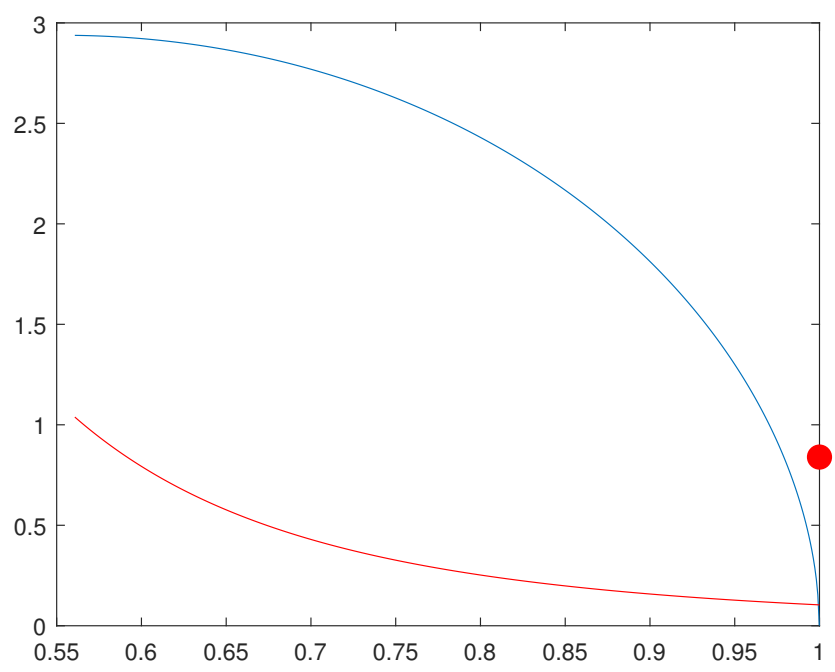

Figure 1. Optimal strategy for the Mobility Player 1 in Blue with optimal strategy for the Terrain Player 2 in Red with a point mass of 0.8394 . Using the values from Table 1 with $b=-0.17$. By equation (17), $\alpha=0.5607$. By equation (18), the value of the game is: Value $=-0.1291$.

\section{SEQUENCE FORM}

\subsection{A simple example using the sequence form}

We consider the following discrete version of the silent duel with detection uncertainty. The game is a zero-sum game between Players 1 and 2. There are $T$ time periods. An initial chance move chooses a time period in 
$\{1 \ldots T\}$ which determines a period $t$ when Player 1 can first act, namely anywhere in time $\{t \ldots T\}$. If $t=T$ then Player 1 has no choice, and must play at $T$.

Subsequently, Player 2, who is neither informed of the chance move nor of the move of Player 1, makes a move in $\{1 \ldots T\}$. The resulting choices, which depend on the chance move and on the choice of the two Players, determine an individual payoff to Player 1, which must be paid by Player 2 .

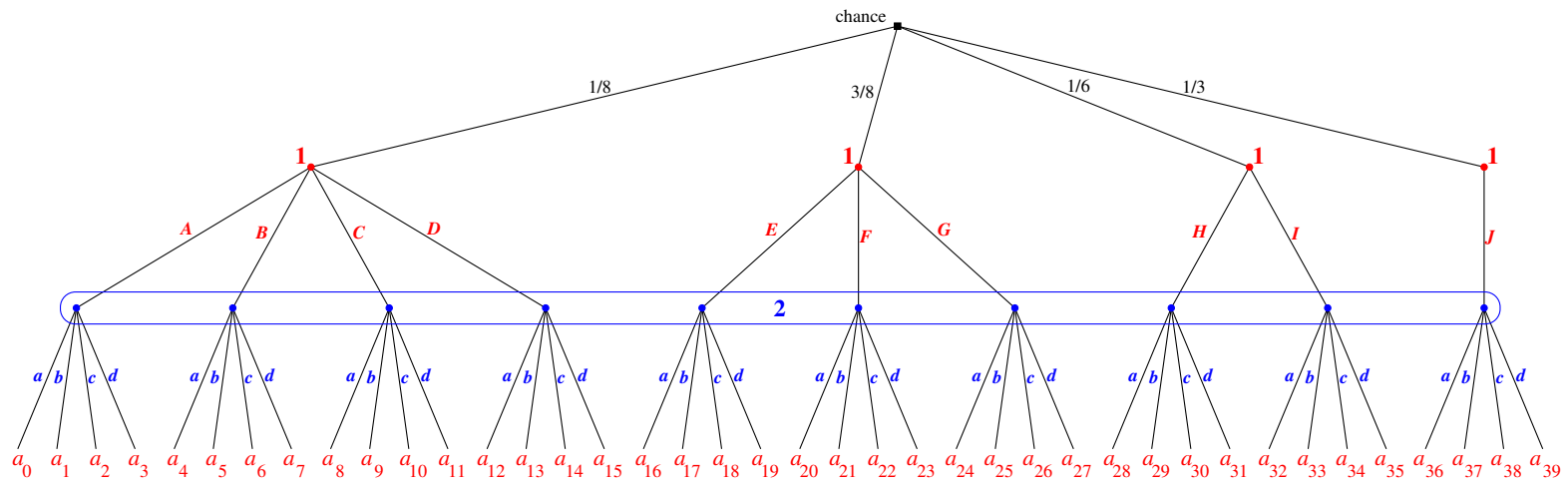

Figure 2. The above figure shows this game in extensive form for $T=4$. The probabilities for the chance move are usually uniform, but we chose them here as $\frac{1}{8}, \frac{3}{8}, \frac{1}{6}, \frac{1}{3}$ to better illustrate how they affect the resulting game matrix.

The game tree has the specific feature of an information set for Player 2, which comprises all her decision nodes, where here she has four moves denoted by $a, b, c, d$. This means that Player 2 is completely uninformed of all previous actions, and by definition makes the same move irrespective of the decision mode she is at. In effect, this means that the two players act simultaneously, except that Player 1 is informed of the initial chance move.

The sequence form $^{5}$ is a way to obtain a compact linear program whose solutions represent the optimal strategies in such game trees with imperfect information. In contrast, perfect information would mean that every information set would contain only a single node, which holds here for Player 1, only.

In this game, the sequence form is particularly simple because each player moves only once, and the "sequences" are just single moves. What we will compute here are in effect behavior strategies which define optimal randomizations over moves rather than over pure strategies, which are combinations of moves. In the present case, Player 1 would already have $4 \times 3 \times 2 \times 1=24$ pure strategies and the corresponding mixed strategy probabilities, whereas we need only $4+3+2+1=10$ behavior probabilities, an obvious gain, particularly for larger $T$. So we just think of sequences as moves of this game.

We introduce variables for the move probabilities, which in this game are the vectors of the variables $x=$ $\left(x_{A}, x_{B}, x_{C}, x_{D}, x_{E}, x_{F}, x_{G}, x_{H}, x_{I}, x_{J}\right)$ for Player 1 and $y=\left(y_{a}, y_{b}, y_{c}, y_{d}\right)$ for Player 2 . These are subject to the constraints $x \geq 0, y \geq 0$, and

$$
x_{A}+x_{B}+x_{C}+x_{D}=1, \quad x_{E}+x_{F}+x_{G}=1, \quad x_{H}+x_{I}=1, \quad x_{J}=1
$$

which we write in matrix notation as $E x=e$ where $e^{T}=\left[\begin{array}{llll}1 & 1 & 1 & 1\end{array}\right]$ and

$$
E=\left(\begin{array}{llllllllll}
1 & 1 & 1 & 1 & 0 & 0 & 0 & 0 & 0 & 0 \\
0 & 0 & 0 & 0 & 1 & 1 & 1 & 0 & 0 & 0 \\
0 & 0 & 0 & 0 & 0 & 0 & 0 & 1 & 1 & 0 \\
0 & 0 & 0 & 0 & 0 & 0 & 0 & 0 & 0 & 1
\end{array}\right)
$$

and

$$
y_{a}+y_{b}+y_{c}+y_{d}=1,
$$

which we write as $F y=f$ with $F=\left[\begin{array}{llll}1 & 1 & 1 & 1\end{array}\right]$ and $f=1$. For the above game we obtain the following linear program (LP) which we explain subsequently. 


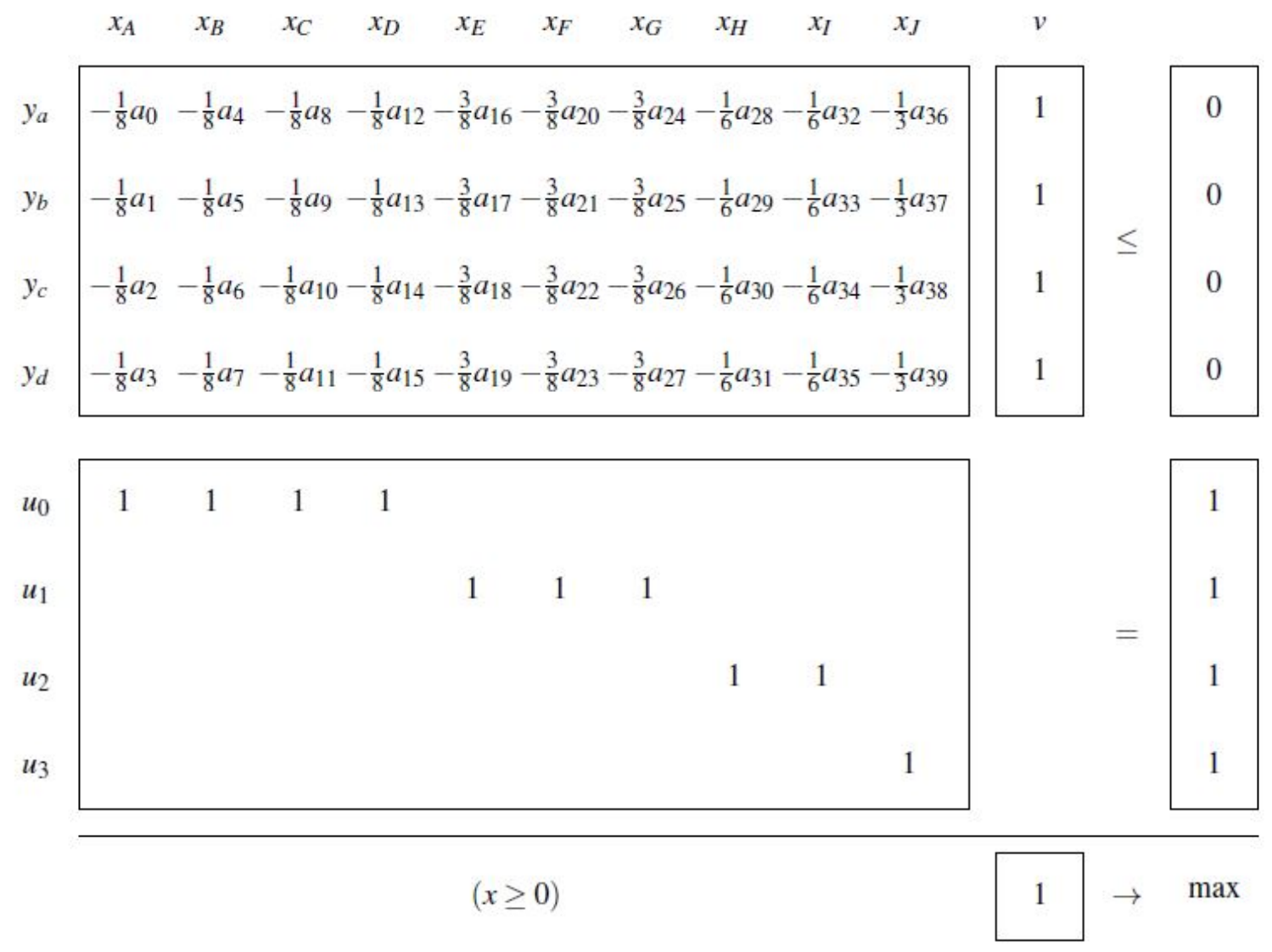

Figure 3. Tucker Diagram showing the free variables $x$ for Player 1 and $y$ for Player 2

The primal variables of this LP written at the top are the vector $x$ subject to the constraint $x \geq 0$ and a single unconstrained variable $v$. Its dual variables, written on the left, are $y \geq 0$ and the unconstrained vector $u=\left(u_{0}, u_{1}, u_{2}, u_{3}\right)$. Only the primal constraints are shown, which have the top part $-A^{T} x+F^{T} v \leq 0$ where $A^{T}$ and $F^{T}$ are the transposed matrices $A$ and $F$. The general form of this LP is

$$
\begin{aligned}
\max _{x, v} & f^{T} v \\
\text { s.t. } \quad-A^{T} x+F^{T} v & \leq 0 \\
E x & =e \\
x & \geq 0
\end{aligned}
$$

Here $A$ is the matrix of payoffs to Player 1 , which in the sequence form is constructed as follows. The columns of $A^{T}$ which are the rows of $A$ are the sequences or moves of Player 1 . This is because Player 1 is normally the row player in a matrix game. The rows of $A^{T}$ which are the columns of $\mathrm{A}$ are the sequences or moves of Player 2. The entries of $A$ are obtained as follows: Consider each leaf or terminal node of the game tree to define a unique path from the root of the game tree. On this path we have

(a) a unique sequence of moves of Player 1, which are single moves here,

(b) a unique sequence of moves of Player 2, which is a single move here,

(c) a product of chance probabilities to reach the leaf, which is a single probability here. 
The resulting entry of $A$ for the row from (a) and column from (b) is the payoff at the leaf to Player 1 multiplied by the product of the chance probabilities from (c). For example, the leaf of the game tree with payoff $a_{29}$ is reached by move $H$ of Player 1 , move $b$ of Player 2, with chance probability $1 / 6$. The corresponding entry in column $H$ and row $b$ of $A^{T}$ is $\frac{1}{6} a_{29}$. In the above LP for our game, the entries are those of the negated matrix $-A^{T}$, hence the minus signs.

In general, not all pairs of sequences lead to a leaf of a game tree where the resulting entry of $A$ must be zero, so that $A$ is in general a sparse matrix. In our example, $A$ is a full matrix, which is due to the fact that Player 2 learns nothing at all about the game state, so that the number of leaves equals the number of matrix entries. In general, there may also be leaves where some player does not move at all, in which case one should introduce the empty sequence $\emptyset$ of moves for such a player. Also, when a player moves more than once, the constraints for the sequences $E x=e$ are more complicated. Finally, for leaves of the game tree where there are no chance moves, the empty product of chance probabilities in 3. above is 1 . Our example is more regularly structured. An optimal solution to the above LP (41) defines a max-min strategy $x$ of Player 1, and its dual solution a min-max strategy $y$ of Player 2.

The derivation of the LP (41) is quickly told. Both players have mixed strategies $x$ and $y$, which for the sequence form are called realization plans. They are played according to the constraints $E x=e$ where $x \geq 0$ and $F y=f$ where $y \geq 0$. For our game these realization plans translate immediately into behavior strategies of the two players. When Player 1 faces $y$, his expected payoff is $x^{T} A y$, which corresponds to the summation over all leaves of the game tree of the corresponding payoffs, multiplied by the respective probabilities of reaching the leaf. This depends on $x, y$, and the chance probabilities. Player 1 tries to maximize his payoff $x^{T} A y$ subject to the constraints $E x=e$ and $x \geq 0$. For fixed $y$, this is a linear program, but if $y$ is a variable, it is not. However, we can consider instead the dual of this LP, which uses a dual vector $u$ of the unconstrained variables, one for each equation in $E x=e$. This dual says:

$$
\begin{array}{ll}
\min _{u} & e^{T} u \\
\text { s.t. } & E^{T} u \geq A y
\end{array}
$$

and it has the same optimal value $e^{T} u$ as the primal value $x^{T} A y$ for a best response $x$ of Player 1 to $y$. Player 2 wants to minimize this amount she has to pay, and the constraints (42) stay an LP even if $y$ is treated as a variable. Note that (42) has the "separated" variables $u$ and $y$ whereas the original problem maximizes a quadratic expression $x^{T} A y$. With $y$ as a vector of variables, (42) becomes

$$
\begin{aligned}
\min _{y, u} & e^{T} u \\
\text { s.t. } \quad-A y+E^{T} u & \geq 0 \\
F y & =f \\
y & \geq 0
\end{aligned}
$$

and it has the same optimal value $e^{T} u$ as the primal value $x^{T} A y$ for a best response $x$ of Player 1 to $y$.

An optimal solution $y, u$ to the LP (43) describes a min-max strategy for Player 2. Importantly, this LP has small dimensions: The number of rows in this LP is the number of sequences of Player 1, i.e. the rows of $A$ plus the number of information sets of Player 2, i.e. the rows of $F$. The number of columns in this LP is the number of sequences of Player 2, i.e. columns of $A$ plus the number of information sets of Player 1, i.e. rows of $E$ and thus columns of $E^{T}$. The dual LP to (43) is just (41) above. An optimal solution $x, v$ to (41) determines a max-min strategy $x$ of Player 1 . The two LPs together determine a pair of optimal behavior strategies $x, y$, i.e. Nash equilibrium of the extensive game.

\subsection{Revisiting Example 2 using the sequence form}

We return now to example 2 in Section 3.2. We use the values from Table 1, except we replace $b=-0.17$. We apply the sequence form to the problem using a grid size of 250. Using linprog in MATLAB, we obtain the results shown in Figure 4. What is shown approximates the analytic result for the Terrain Player (Player 2). We 
see the method is approximating a point mass at the end of the interval, because of the sharp increase in slope. The sum of the discrete probabilities at the end of the interval is 0.8437 , which compares to the analytic value of the point mass, 0.8394 .

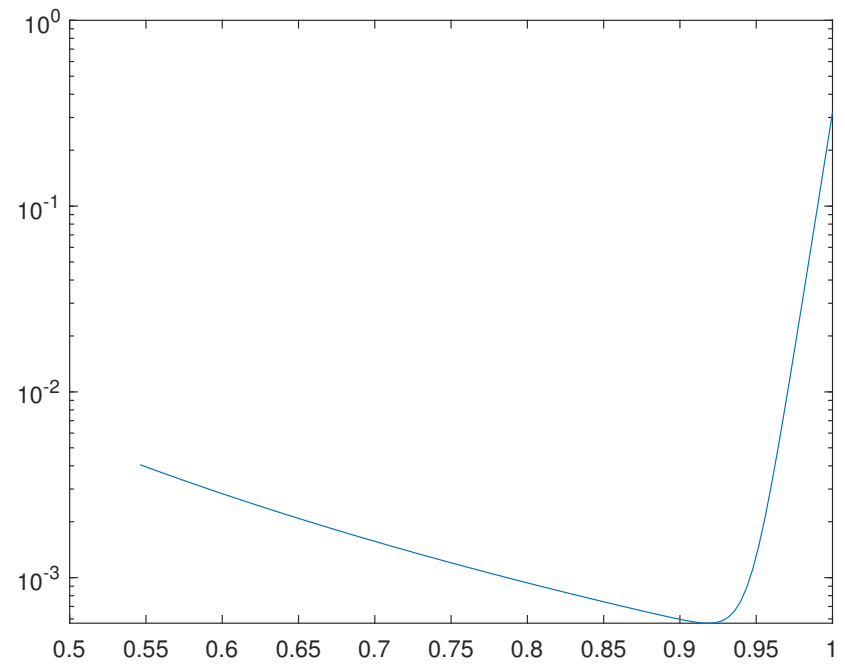

Figure 4. Using the values from Table 1 with $b=-0.17$ we apply the sequence form. As shown in Section 3.2 we should have a point mass of 0.8394 . Here we show the numerical results of the optimal strategy for the Terrain Player (Player 2) in blue, with a point mass approximately 0.8437. By equation (17), $\alpha=0.5607$. Here we show $\alpha=0.5462$. By equation (18), the value of the game is:Value $=-0.1291$. Here we show Value $=-0.1281$.

\section{REFERENCES}

[1] Bednarz, D. and Muench, P., "Mobility versus terrain: a game theoretic approach," in [Unmanned Systems Technology XVIII], Proc. SPIE 9837 (2016).

[2] Scarf, H. E. and Shapley, L. S., "Games with partial information," RAND Corporation Papers P-797 (1956).

[3] Shapley, L. S., "Stochastic games," Proceedings of the National Academy of Sciences 39(10), 1095-1100 (1953).

[4] David Bednarz, P. M. and Krupansky, N. A., "A game of timing with detection uncertainty," in [Unmanned Systems Technology XX], Proc. SPIE 10640 (2018).

[5] von Stengel, B., "Efficient computation of behavior strategies," Games and Economic Behavior 14, 220-246 (1996). 